\title{
Variability-Conscious Circuit Designs for Low-Voltage Memory-Rich Nano-Scale CMOS LSIs
}

\author{
Kiyoo Itoh \\ Fellow, Central Research Laboratory, Hitachi, Ltd. \\ 1-280 Higashi-Koigakubo, Kokubunji, Tokyo 185-8601, Japan \\ Tel.: +81-42-323-1111 \\ kiyoo.itoh.pt@hitachi.com
}

\begin{abstract}
Low-voltage scaling limitations of nanoscale CMOS LSIs are one of the major problems in the nanoscale era because they cause the evermore-serious power crises with device scaling. The problems stem from two unscalable device parameters: The first is the high value of the lowest necessary threshold voltage $\mathrm{Vt}$ (that is, $\mathrm{Vt} 0$ ) of MOSFETs needed to keep the subthreshold leakage low. The second is the variation in $\mathrm{Vt}$ (that is, $\Delta \mathrm{Vt}$ ), that becomes more prominent in the nanoscale era. The $\Delta \mathrm{Vt}$ caused by the intrinsic random dopant fluctuation is the major source of various $\Delta \mathrm{Vt}$ components. It increases with device scaling and thus intensifies various detrimental effects such as variations in speed and/or the voltage margins of circuits. Due to such inherent features of $\mathrm{Vt} 0$ and $\Delta \mathrm{Vt}$, the operating voltage VDD is facing a $1-\mathrm{V}$ wall in the $65-\mathrm{nm}$ generation, and is expected to rapidly increase with further scaling of bulk MOSFETs, thereby worsening the power crisis. To reduce VDD, the minimum operating voltage Vmin, as determined by $\mathrm{Vt} 0$ and $\Delta \mathrm{Vt}$, must be reduced.

In this talk the Vmin of memory-rich nanoscale CMOS LSIs is investigated in an effort to reduce to below $0.5 \mathrm{~V}$ through variability-conscious device and circuit designs. First, Vmin, as a methodology to evaluate the low-voltage potential of MOSFETs, is proposed on the basis of a tolerable speed variation. Second, Vmins of the logic, SRAM, and DRAM blocks are compared, and the SRAM block comprising the six-transistor (6-T) cell turns out to be particularly problematic because it has the highest Vmin. Third, new devices, such as a fully-depleted structure (FD-SOI) and fin-type structure (FinFET) as $\triangle \mathrm{Vt}$-immune MOSFETs, are investigated to further reduce the Vmins of the above-described blocks. Also investigated are new circuits to reduce Vmin of each block. For example, for the logic block, new dual-Vt0 and dual-VDD dynamic circuits enable the powerdelay product to be reduced to 0.09 at a $0.2-\mathrm{V}$ supply owing to gate-source reverse biasing. For the SRAM block, repair techniques, shortening the data line, upsizing the MOSFETs, control of the common-source line or the word line of the cell, and even the 8-T cell reduce the Vmin. For the DRAM block, if combined with FinFET DRAM cells, a dynamic sense amplifier minimizes the Vt0 and thus Vmin.

Finally, it is concluded that such variability-conscious circuit designs should lead to the achievement of $0.5-\mathrm{V}$ nanoscale LSIs, if relevant devices and fabrication processes are successfully developed.
\end{abstract}

